\title{
Clinical research and factors associated with prolonged duration of viral shedding in patients with COVID-19
}

\section{Di Tian}

Beijing Ditan Hospital

\section{Lin Wang}

Beijing Ditan Hospital

\section{Xiankun Wang}

Beijing Ditan Hospital

\section{Ziruo Ge}

Beijing Ditan Hospital

\section{Shuping Cui}

Beijing Ditan Hospital

Yanli Xu

Beijing Ditan Hospital

\section{Rui Song}

Beijing Ditan Hospital

\section{Meihua Song}

Beijing Ditan Hospital

\section{Bing Han}

Beijing Ditan Hospital

\section{Wei Zhang}

Beijing Ditan Hospital

Dawei Tan

Beijing Ditan Hospital

\section{Xingang Li}

Beijing Ditan Hospital

Yang Zhou

Beijing Ditan Hospital

Sujuan Zhang

Beijing Ditan Hospital

\section{Li Yang}

Beijing Ditan Hospital

\section{Peipei Meng}


Beijing Ditan Hospital

\section{Le Sun}

Beijing Ditan Hospital

\section{Xingwang Li}

Beijing Ditan Hospital

Zhihai Chen ( $\square$ chenzhihai0001@126.com )

Beijing Ditan Hospital, Capital Medical University

\section{Research article}

Keywords: COVID-19, Clinical research, viral shedding

Posted Date: June 1st, 2020

DOl: https://doi.org/10.21203/rs.3.rs-29818/v1

License: (c) (i) This work is licensed under a Creative Commons Attribution 4.0 International License. Read Full License 


\section{Abstract \\ Background}

Towards the end of December 2019, the Wuhan health commission declared an outbreak of clusters of pneumonia in patients. Sequencing indicated that this disease (COVID-19) was caused by a novel coronavirus (SARS-CoV-2). The outbreak of COVID-19 is currently still underway.

\section{Methods}

We recruited 75 SARS-CoV-2 infected patients admitted to the Center of Infectious Disease division 2 of Beijing Ditan Hospital from Jan 20 to Mar 20, 2020. Epidemiological, demographic, clinical, radiological features, laboratory data were analyzed.

\section{Results}

Of the 75 patients, $42(56 \%)$ patients were male and 33(44\%) patients were female. The mean age of all patients was $41.5 \pm 19.4$ years. Male patients were more likely to become severe. There were 9 family clusters accounted for 44 patients. Patients classified as being severe had a higher frequency of fever upon admission than patients classified as moderate cases. For moderate patients, the median duration of viral shedding was $25(9.5,42)$ days (range $1-63$ days) from the first positive nucleic acid test compared to $14(9,21.25)$ days (range $2-62$ days) for severe cases. The difference between the two groups was statistically significant $(p=0.041)$. Cox regression analyses indicated that disease status and CRP were the factors that affect the duration of viral shedding. Virus clearance was significantly faster in severe patients compared to moderate patients $(p=0.011)$, and patients with CRP range in 2-10 times higher than upper limit of normal value had longer duration of viral shedding $(p=0.012)$. CRP and CD $4+T$ lymphocyte was negative correlated, and the relationship between CRP and CD $4+T$ lymphocyte was statistically significant $(P=0.003)$, with a correlation coefficient of -0.564 . During the second week following the onset of illness, severe cases had higher WBC, NEU and CRP, but lower LYM, MON and EOS as compared with moderate cases (all $\mathrm{P}<0.05$ ). Severe cases still had lower lymphocyte counts and higher CRP than moderate cases in the third week.

\section{Conclusions}

Viral clearance was significantly prolonged in moderate patients, and those CRP in 2-10 times higher than upper limit of normal value. Immune response may affect the duration of viral shedding. Severe cases had a persistence lower lymphocyte count and higher CRP than moderate cases.

\section{Background}


Since December 2019, there has been a series of unexplained cases of pneumonia reported in Wuhan, China. On 12 January 2020, the World Health Organization (WHO) tentatively named this new virus the 2019 novel coronavirus (2019-nCoV). On 30 January 2020, the WHO announced that the 2019unced 2019, thwas a public health emergency of international concern. On 11 February 2020, the WHO formally named the disease triggered by 2019-nCoV as coronavirus disease 2019 (COVID-19). On the same day, the coronavirus study group of the International Committee on Taxonomy of Viruses named 2019-nCoV as severe acute respiratory syndrome coronavirus 2 (SARS-CoV-2) ${ }^{[1]}$.

SARS-CoV-2 has spread to other geographic locationsCE13F53A2ED\}ered by 2019-nCoV as coronavirus disease transmission ${ }^{[2]}$. So far, there have been 561 laboratory-confirmed patients reported in Beijing. More than two months have passed since patients with COVID-19 were first admitted into hospitals. A portion of the patients showed that their nucleic acid did not turn negative for a significant amount of time, and a few of them had positive conversion results after two negative nucleic acid tests.

In this study, we analyzed epidemiological, clinical, laboratory, etiologic detection, and radiological characteristics of patients confirmed to have SARSatientsinfection.

\section{Methodspatients}

This is a prospective and observational cohort study on the COVID-19. We enrolled patients from a singlecenter, Beijing Ditan Hospital of Capital Medical University. We investigated the demographic features, clinical manifestation, laboratory, and imaging results of 75 patients, who were confirmed to have SARSCoV-2 infection by real-time PCR from January through March 2020 in Ditan hospital. This study complied with the principles of the Declaration of Helsinki. Informed consent was obtained from all patients or their immediate relatives for sample and data collection and their use for research.

Case confirmation for SARS-CoV-2 infection is based on laboratory diagnosis through detection of the virus by PCR (Shanghai BioGerm Medical Technology Co. LTD) in the specimens obtained from patients, regardless of the presence of clinical signs and symptoms.

\section{Treatment}

All the patients received supportive measures, including rest, nutritional support, and electrolyte balance maintenance. Most patients took lopinavir/ritonavir tablets orally and recombinant human interferonalpha nebulizer by inhalation. For those patients with severe COVID-19, glucocorticoid and human gamma globulin were administered.

\section{Data collection and definitions}

Demographic and clinicopathological data were collected. Blood samples were collected upon admission to the hospital and throughout the disease course. For clinical data collection, we designed a medical record form and online database that contains information on epidemiologic, clinical manifestations, 
physical examinations, and laboratory parameters. Demographic factors and dates of onset were included. All data were entered by trained study staff.

The date of disease onset was defined as the day fever or cough was noticed. These patients were further divided into four subgroups based on the aforementioned case definitions, which included the groups of mild, moderate, severe, and critical cases, for analyses. Patients with mild symptoms and without signs of pneumonia by imaging were considered as mild cases. Patients with fever and respiratory symptoms with radiological findings of pneumonia were classified as moderate cases. Patients who met one of the following criteria were considered as severe COVID-19 (or SARI) cases in our study: 1) dyspnea with the respiratory rates > 30/min; 2) hypoxemia with $\mathrm{O}_{2}$ Saturation < 93\%; 3) PaO2 / FiO2 < 300 mmHg.4) respiratory failure and requiring mechanical ventilation; 5) shock; 6) with organ failure that required ICU care.

\section{Statistical analysis}

All statistical analyses were conducted with SPSS 25.0 (IBM, Armonk, NY, USA). Graphpad prism 8 was used to conduct the correlation figure. Continuous variables are presented as mean \pm standard deviation or medians with interquartile ranges, while categorical variables are presented as frequencies or percentages of events. The two-sample t-test or Mann-Whitney $U$ test was used to determine the relationship between moderate and severe for continuous data. The Pearson chi-square and Fisher's exact tests were used to compare differences in proportion between groups, as appropriate. Independent factors were identified by univariable and multivariable Cox regression analyses for predicting viral shedding time.

\section{Ethics statement}

This study was approved by the local Ethics Committee of the lead center, Beijing Ditan Hospital, Capital Medical University (YAN 2020-015-01) and complied with the principles of the Declaration of Helsinki.

\section{Results}

\section{Demographics and epidemiological history}

Of the 75 patients, $42(56 \%)$ patients were male and $33(44 \%)$ patients were female. The mean age of all patients was $41.5 \pm 19.4$ years, ranging from $0.8-88$ years. $5(7 \%)$ patients were $0-6$ years of age, and $4(5 \%)$ patients were 66 years old or older. $10(13 \%)$ patients were considered to be mild cases. $45(60 \%)$ patients were classified as moderate cases, while 20(27\%) patients met the severe criteria. 4(5\%) patients required invasive mechanical ventilation. By the end of $12 \mathrm{Apr}$, all 75 patients had been discharged.

26 men and 19 women were classified as being moderate cases, while 14 men and 6 women were classified as being either severe cases. There was a statistical difference between the two groups, and male patients were more likely to become severe. 
There were 30 patients with a history of travel to or residence in Wuhan and its surrounding areas 14 days prior to the onset of the disease. 46 patients had contact with patients who had a fever or respiratory symptoms from Wuhan and its surrounding areas within 14 days before the onset of the disease. 9 family clusters accounted for 44 patients in total. (Table 1)

\section{Clinical manifestations}

Upon admission, the main clinical manifestations observed in patients included fever, fatigue, cough, expectoration, and muscle aches. Headache, dizziness, anorexia, and diarrhea were found in a few cases. Those patients classified as being severe had a higher frequency of fever upon admission than patients classified as moderate cases. The median time from illness onset to admission was 7.0 days. (Table 2 )

\section{CT findings}

All patients underwent chest computed tomography on admission and 87\% (65/75) manifested as pneumonia. The most common patterns observed on chest computed tomography were ground glass opacity and bilateral patchy shadowing.

In the early stage of disease onset, imaging showed multiple small patchy shadows and interstitial changes, apparent in the outer lateral zone of the lungs. As the disease progressed, imaging then showed multiple ground glass opacities and infiltration in both lungs. In severe cases, pulmonary consolidation may occur.

\section{Etiologic detection}

For moderate patients, the median duration of viral shedding was $25(9.5,42)$ days (range $1-63$ days) from the first positive nucleic acid test compared to $14(9,21.25)$ days (range $2-62$ days) for severe cases. The difference between the two groups was statistically significant $(p=0.041) .8$ patients showed positive conversion after two negative nucleic acid tests, and all of them had no manifestation.(Fig. 1)

\section{Laboratory assessment}

During the second week following the onset of illness, severe cases had higher WBC (white blood cell), NEU (neutrophil) and CRP (C-reactive protein), but lower LYM (lymphocyte), MON (monocyte) and EOS (eosinophil) as compared with moderate cases (all $\mathrm{P}<0.05$ ). (Table 3 )

In the third week from the onset of illness, severe cases still had lower lymphocyte counts and higher CRP than moderate cases ( $p=0.001$ and 0.003 , respectively), with a statistical difference between the two groups. (Table 3)

Univariate Cox regression analyses revealed that disease status, elevated WBC, elevated neutrophil, elevated levels of CRP were the factors for shortening the duration of viral shedding. The above variables were used for multivariate Cox regression analyses. The results indicated that disease status and CRP 210 times higher than the upper limit of normal value were the independent factors that affect the duration of viral shedding. Virus clearance was significantly faster in severe patients compared to moderate 
patients $(\mathrm{p}=0.011)$, and patients with CRP range in 2-10 times higher than upper limit had longer duration of viral shedding $(p=0.012)$. (Fig. 2$)$ The detailed results of the multivariate analysis are shown in Table 4.

CRP and $C D 4^{+} T$ lymphocyte was negative correlated, and the relationship between CRP and $C D 4^{+} T$ lymphocyte was statistically significant $(P=0.003)$, with a correlation coefficient of -0.564 . (Fig. 3 )

\section{Discussion}

In our study, we report on a cohort of 75 patients with laboratory-confirmed SARS-CoV-2 infection. They had a history of travel to or residence in Wuhan and its surrounding areas or had been in contact with patients who had a fever or respiratory symptoms from Wuhan and its surrounding areas within 14 days before the onset of the disease. There were 9 family clusters among 75 patients, indicated human to human transmission. In China, beginning on 24 January 2020, hundreds of millions of people traveled to their hometowns or overseas for the Chinese New Year holiday ${ }^{[3]}$. In a study, 133 patients from more than 20 families were infected. Similar to Wuhan, the infection of COVID-19 in Beijing was of clustering onset as well $[4]$.

In our study, there were more male patients in severe cases than in moderate cases, and indicating male patients are more likely to become severe. Chen Tao et al ${ }^{[5]}$, previously concluded that male sex was more predominant in deceased patients $(83 ; 73 \%)$ than in recovered patients $(88 ; 55 \%)$.

A research demonstrated that the Asian males has an extremely large number of ACE2-expressing cells in the lung ${ }^{[6]}$. Another study demonstrated that the East Asian populations have higher ACE2 expression in tissues, which may suggest different susceptibility or response to 2019-nCoV/SARS-CoV-2 from different populations under similar conditions ${ }^{[7]}$.

Our study also found that five patients were $0-6$ years of age, all of which were mild cases. Our results indicate that children might be less likely to become infected or, if infected, may show milder symptoms. According to the current conditions of the reported cases, the infected children mainly belong to family cluster cases or had close contact with infected cases $^{[8]}$.

According to our study, the main clinical manifestations included fever, fatigue, cough, expectoration, and muscle aches, all of which are similar to those reported by other research ${ }^{[9-12]}$. Our study revealed that more patients in severe cases had fever upon admission than patients in moderate cases.

Our study turned out that the median time from illness onset to admission was 7.0 days. So, we compared laboratory parameters of two group in 2nd week and 3rd week after onset of illness, and severe cases had lower lymphocyte counts and higher CRP than moderate cases during. Similar to Chen Tao's research, deceased patients had persistent and more severe lymphopenia than recovered patients ${ }^{[5]}$. 
Our study showed the median duration of viral shedding in moderate patients was 25 days, which was much longer than that of severe cases (14 days). In another study of 191 patients with COVID-19, Fei Zhou et $a^{[13]}{ }^{[1}$ ound that the median time of virus clearance was 20 days, and the longest time was 37 days. For SARS-CoV, the median (range) duration of viral excretion was 21 (14-52) days for sputum samples and $27(16-126)$ days for stool specimens ${ }^{[14]}$. Another study concluded that peak viral loads of SARS-CoV were reached at $12-14$ days of illness ${ }^{[15]}$.

Cox regression analyses indicated that patients in moderate group and had CRP 2-10 times higher than upper limit had prolonged duration of viral shedding, and CRP and CD4 ${ }^{+} T$ lymphocytes were negative correlated. We concluded that immune response may play an important role in viral shedding. Yun Ling et $\mathrm{al}^{[16]}$ used multiple linear regression model, the analysis showed the CD4 + T lymphocyte counts may help predict the the duration of viral RNA detection in patients'stool, and the lower the absolute value of $\mathrm{CD} 4^{+} \mathrm{T}$ lymphocytes before treatment, the longer duration of virus clearance.

Some patients had positive conversion results after two negative nucleic acid tests. Recently, 2019-nCoV has been detected in human clinical specimens by next-generation sequencing, real-time RT-PCR, cell culture, and electron microscopy. Specimens from the upper respiratory tract are more likely to turn out false negative. In order to increase the positive rate of nucleic acid detection, it is recommended to take as much sputum as possible.

Our study has some limitations. First, we are not the first hospital for patients. Most of the patients we admit have been transferred from other hospitals following diagnosis. Thus, we lack the data during the first week of infection. Second, the estimated duration of viral shedding is limited by the frequency of respiratory specimen collection and the lack of quantitative viral RNA detection. Third, we lack the data for stool specimens.

\section{Declarations}

\section{Ethics approval and consent to participate}

This study was approved by the local Ethics Committee of the lead center, Beijing Ditan Hospital, Capital Medical University (YAN2020-015-01) and complied with the principles of the Declaration of Helsinki. All participants provided written informed consent.

\section{Consent for publication}

Not applicable

\section{Availability of data and materials}

All data generated or analysed during this study are included in this published article. 
The authors declare that they have no competing interests.

\section{Funding}

Supported by Beijing Municipal Administration of Hospitals Clinical Medicine Development of Special Funding Support (XMLX201502 and XMLX201602), and National Science and Technology Major Project of China (2018ZX09711003).

\section{Authors' contributions}

Di Tian and Lin Wang carried out the studies, participated in collecting data, and drafted the manuscript. Di Tian and Xiankun Wang performed the statistical analysis and participated in its design. Di Tian and Lin Wang participated in acquisition, analysis, or interpretation of data and draft the manuscript. All authors read and approved the final manuscript.

\section{Acknowledgements}

The authors would like to thank all study participants who were enrolled in this study.

\section{References}

1. Sun P, Lu X, Xu C, et al. Understanding of Covid-19 Based On Current Evidence. J Med Virol. 2020.

2. Nishiura $\mathrm{H}$, Jung SM, Linton NM, et al. The Extent of Transmission of Novel Coronavirus in Wuhan, China, 2020. J Clin Med. 2020; 2.

3. Li X, Zai J, Wang X, Li Y. Potential of Large "First Generation" Human-to-Human Transmission of 2019-Ncov. J Med Virol. 2020;4:448-54.

4. Tian S, Hu N, Lou J, et al. Characteristics of Covid-19 Infection in Beijing. J Infect. 2020;4:401-6.

5. Chen T, Wu D, Chen H, et al. Clinical Characteristics of 113 Deceased Patients with Coronavirus Disease 2019: Retrospective Study. BMJ. 2020; m1091.

6. Zhao Y, Zhao Z, Wang Y. Single-Cell Rna Expression Profiling of Ace2, the Putative Receptor of Wuhan 2019-Ncov. 2020.

7. Cao Y, Li L, Feng Z, et al. Comparative Genetic Analysis of the Novel Coronavirus (2019-Ncov/SARSCov-2) Receptor Ace2 in Different Populations. Cell Discov. 2020; 11.

8. Chen ZM, Fu JF, Shu Q, et al. Diagnosis and Treatment Recommendations for Pediatric Respiratory Infection Caused by the 2019 Novel Coronavirus. World J Pediatr. 2020.

9. Huang C, Wang Y, Li X, et al. Clinical Features of Patients Infected with 2019 Novel Coronavirus in Wuhan, China. Lancet. 2020;10223:497-506.

10. Wang W, Tang J, Wei F. Updated Understanding of the Outbreak of 2019 Novel Coronavirus (2019Ncov) in Wuhan, China. J Med Virol. 2020;4:441-7. 
11. Wang D, Hu B, Hu C, et al. Clinical Characteristics of 138 Hospitalized Patients with 2019 Novel Coronavirus-Infected Pneumonia in Wuhan, China. JAMA. 2020.

12. Zhang JJ, Dong X, Cao YY, et al. Clinical Characteristics of 140 Patients Infected with SARS-Cov-2 in Wuhan, China. Allergy. 2020.

13. Zhou F, Yu T, Du R, et al. Clinical Course and Risk Factors for Mortality of Adult Inpatients with Covid19 in Wuhan, China: A Retrospective Cohort Study. Lancet. 2020;10229:1054-62.

14. Liu W, Tang F, Fontanet A, et al. Long-Term SARS Coronavirus Excretion From Patient Cohort, China. Emerg Infect Dis. 2004;10:1841-3.

15. Cheng PK, Wong DA, Tong LK, et al. Viral Shedding Patterns of Coronavirus in Patients with Probable Severe Acute Respiratory Syndrome. Lancet. 2004;9422:1699-700.

16. Ling Y, Xu SB, Lin YX, et al. Persistence and Clearance of Viral Rna in 2019 Novel Coronavirus Disease Rehabilitation Patients. Chin Med J (Engl). 2020;9:1039-43.

\section{Tables}

Table 1 Comparison of demographics and epidemiological features in moderate and severe cases of COVID-19

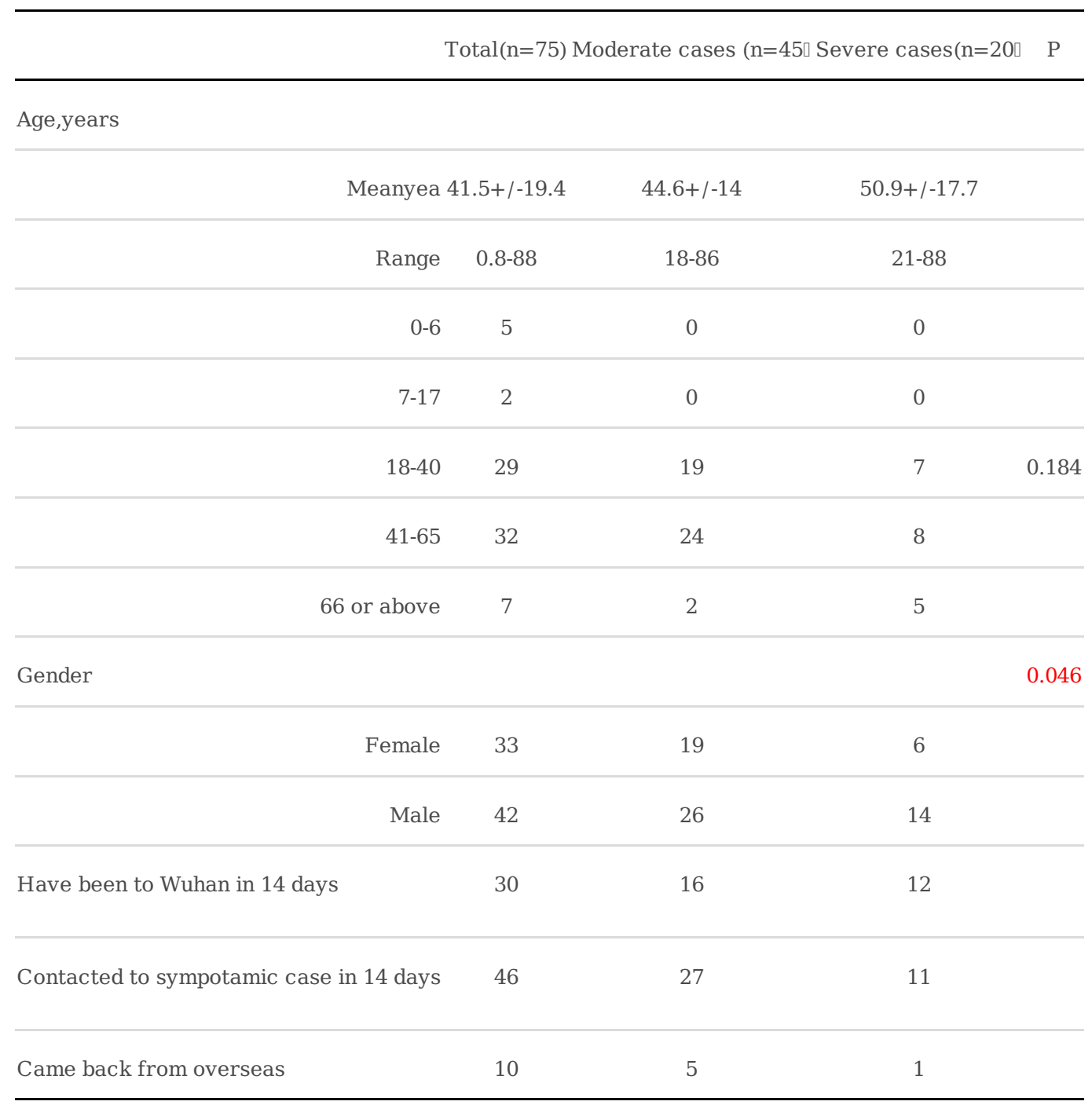


Table 2 Comparison of clinical symptoms in moderate and severe cases of COVID-19

\begin{tabular}{|c|c|c|c|}
\hline & Moderate cases $(n=45 \square$ & Severe cases $(n=20 \square$ & $\mathrm{p}$ value \\
\hline Fever & 20 & 18 & 0.001 \\
\hline Fatigue & 15 & 9 & 0.412 \\
\hline Cough & 26 & 16 & 0.1 \\
\hline Expectoration & 14 & 9 & 0.4 \\
\hline Shortness of breath & 2 & 2 & 0.581 \\
\hline Chest distress & 6 & 2 & 1 \\
\hline Sore throat & 9 & 1 & 0.156 \\
\hline Headache & 11 & 3 & 0.521 \\
\hline Muscle ache & 9 & 8 & 0.127 \\
\hline Diarrhoea & 3 & 2 & 0.639 \\
\hline Anorexia & 5 & 4 & 0.44 \\
\hline Dizziness & 7 & 4 & 0.725 \\
\hline
\end{tabular}

Table 3 Comparison of laboratory parameters in moderate and severe cases of COVID-19 in $2^{\text {nd }}$ week and $3^{\text {rd }}$ week after onset of illness 


\begin{tabular}{lllllll}
\hline & & $2 n d$ week & & & 3rd week \\
& Moderate cases $(\mathrm{n}=45)$ & Severe cases $(\mathrm{n}=20)$ & $\mathrm{P}$ & Moderate cases $(\mathrm{n}=45)$ & Severe cases $(\mathrm{n}=20)$ & $\mathrm{P}$ \\
\hline $\mathrm{WBC}\left(10^{9} / \mathrm{L}\right)$ & $4.43(3.83,4.91)$ & $5.35(3.91,7.20)$ & 0.021 & $4.86(4.40,6.52)$ & $6.30(4.23,8.43)$ & 0.157 \\
\hline $\mathrm{NEU}\left(10^{9} / \mathrm{L}\right)$ & $2.53(2.21,3.24)$ & $4.11(2.62,5.94)$ & 0 & $3.16(2.30,4.05)$ & $5.05(2.71,6.65)$ & 0.013 \\
\hline $\operatorname{LYM}\left(10^{9} / \mathrm{L}\right)$ & $1.37(1.11,1.56)$ & $1.03(0.66,1.23)$ & 0.001 & $1.62(1.35,1.92)$ & $0.97(0.78,1.57)$ & 0.001 \\
\hline $\operatorname{MON}\left(10^{9} / \mathrm{L}\right)$ & $0.31(1078$ & $0.21(1078$ & 0.001 & $0.35(0.27,0.42)$ & $0.36(0.23,0.50)$ & 0.732 \\
\hline $\operatorname{EOS}\left(10^{9} / \mathrm{L}\right)$ & $0.04(0.02,0.08)$ & $0.005(0.00,0.02)$ & 0 & $0.08(0.05,0.15)$ & $0.06(0.04,0.12)$ & 0.402 \\
\hline $\operatorname{CRP}(\mathrm{mg} / \mathrm{L})$ & $11.95(3.25,38.90)$ & $46.55(13.70,96.65)$ & 0.003 & $2.70(1.25,7.60)$ & $13.3(4.70,43.80)$ & 0.003 \\
\hline
\end{tabular}

Table 4 Univariate and multivariate factors associated with the duration of viral shedding in COVID-19 patients 


\begin{tabular}{|c|c|c|c|c|c|c|c|c|c|c|}
\hline & \multicolumn{5}{|c|}{ Univariable analysis } & \multicolumn{5}{|c|}{ Multivariable analysis } \\
\hline & \multirow[t]{2}{*}{$ß$} & \multirow[t]{2}{*}{$\mathrm{P}$} & \multirow[t]{2}{*}{ HR } & \multicolumn{2}{|c|}{ 95\%CI for HR } & \multirow[t]{2}{*}{$\beta$} & \multirow[t]{2}{*}{$\mathrm{P}$} & \multirow[t]{2}{*}{$\mathrm{HR}$} & \multicolumn{2}{|c|}{ 95\% CI for HR } \\
\hline & & & & lower & upper & & & & lower & upper \\
\hline gender & 0.458 & 0.147 & 1.581 & 0.851 & 2.937 & & & & & \\
\hline age & -0.382 & 0.212 & 0.682 & 0.374 & 1.243 & & & & & \\
\hline \multicolumn{11}{|l|}{ WBC } \\
\hline $\mathrm{WBC} 1\left(<4 \times 10^{9} / \mathrm{L}\right)$ & 0.199 & 0.539 & 1.221 & 0.647 & 2.303 & & & & & \\
\hline $\operatorname{WBC} 2\left(>10 \times 10^{9} / \mathrm{L}\right)$ & 1.596 & 0.034 & 4.933 & 1.131 & 21.514 & & & & & \\
\hline \multicolumn{11}{|l|}{ NEU } \\
\hline $\mathrm{N} 1\left(<2 \times 10^{9} / \mathrm{L}\right)$ & 0.051 & 0.908 & 1.052 & 0.443 & 2.498 & & & & & \\
\hline $\mathrm{N} 2\left(>8 \times 10^{9} / \mathrm{L}\right)$ & 1.533 & 0.005 & 4.63 & 1.577 & 13.59 & & & & & \\
\hline LYM & -0.006 & 0.986 & 0.994 & 0.53 & 1.864 & & & & & \\
\hline EOS & -0.097 & 0.744 & 0.908 & 0.508 & 1.621 & & & & & \\
\hline \multicolumn{11}{|l|}{ CRP } \\
\hline CRP1(10-50)mg/L & -0.654 & 0.058 & 0.52 & 0.264 & 1.024 & -0.926 & 0.012 & 0.396 & 0.192 & 0.818 \\
\hline CRP2(51-100mg/L) & 0.266 & 0.525 & 1.304 & 0.574 & 2.963 & -0.267 & 0.575 & 0.766 & 0.301 & 1.946 \\
\hline CRP3(>100mg/L) & 1.653 & 0.012 & 5.224 & 1.437 & 18.993 & 1.158 & 0.094 & 3.184 & 0.822 & 12.331 \\
\hline group & 0.771 & 0.013 & 2.162 & 1.179 & 3.965 & 0.94 & 0.011 & 2.56 & 1.245 & 5.263 \\
\hline
\end{tabular}

Figures 


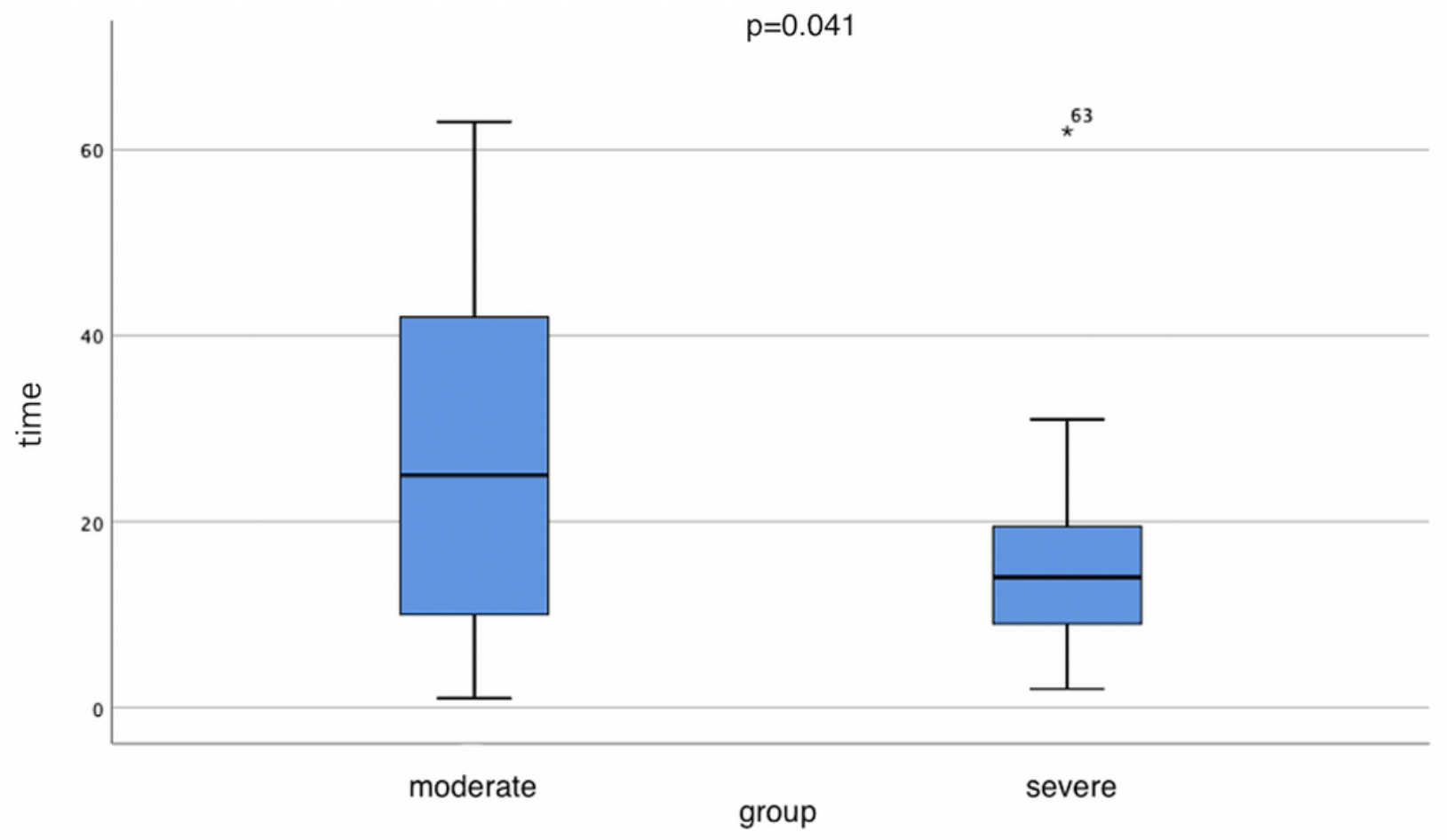

Figure 1

Comparison of duration of viral shedding between two group. 

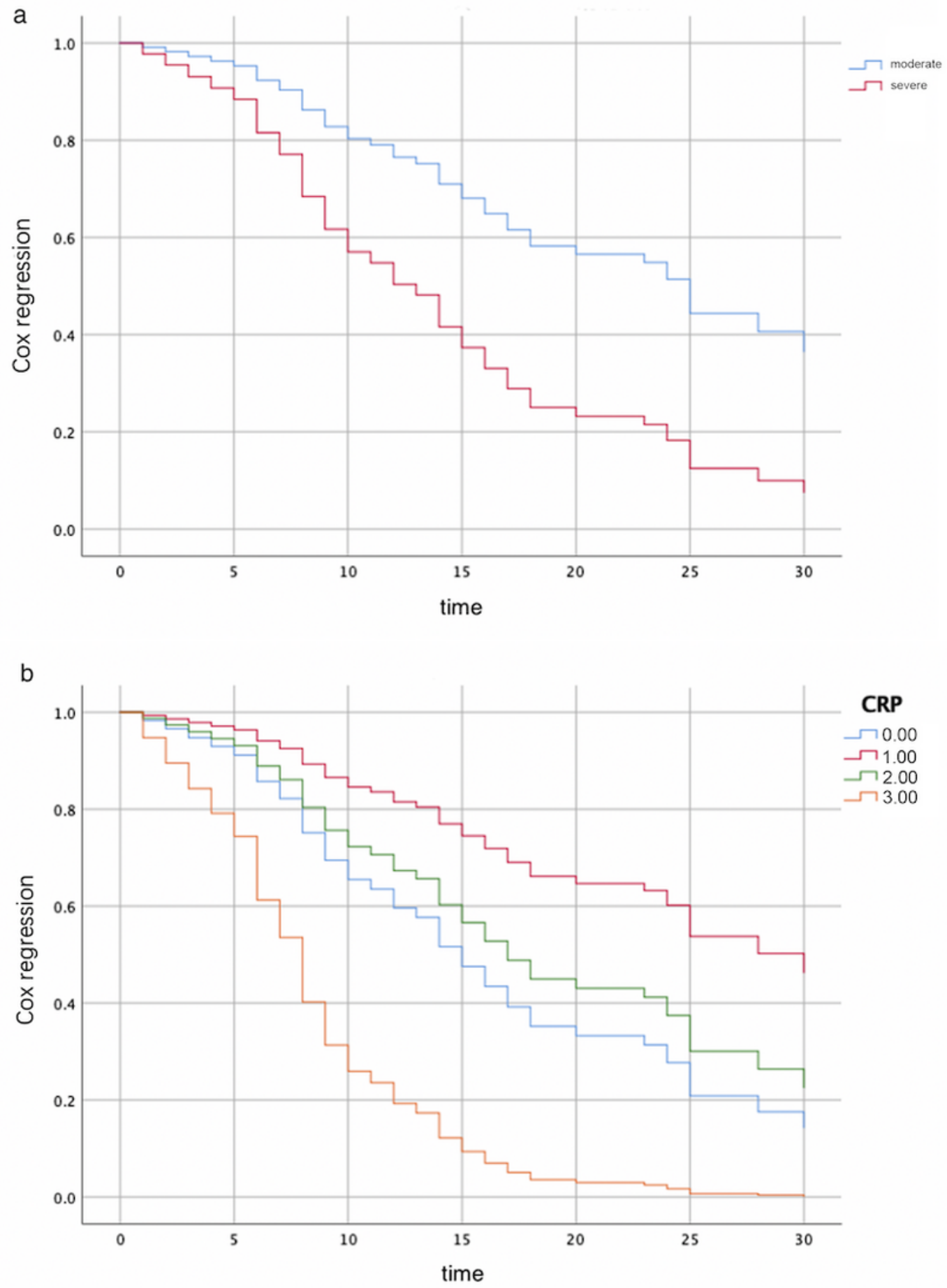

Figure 2

Cox regression analysis. 


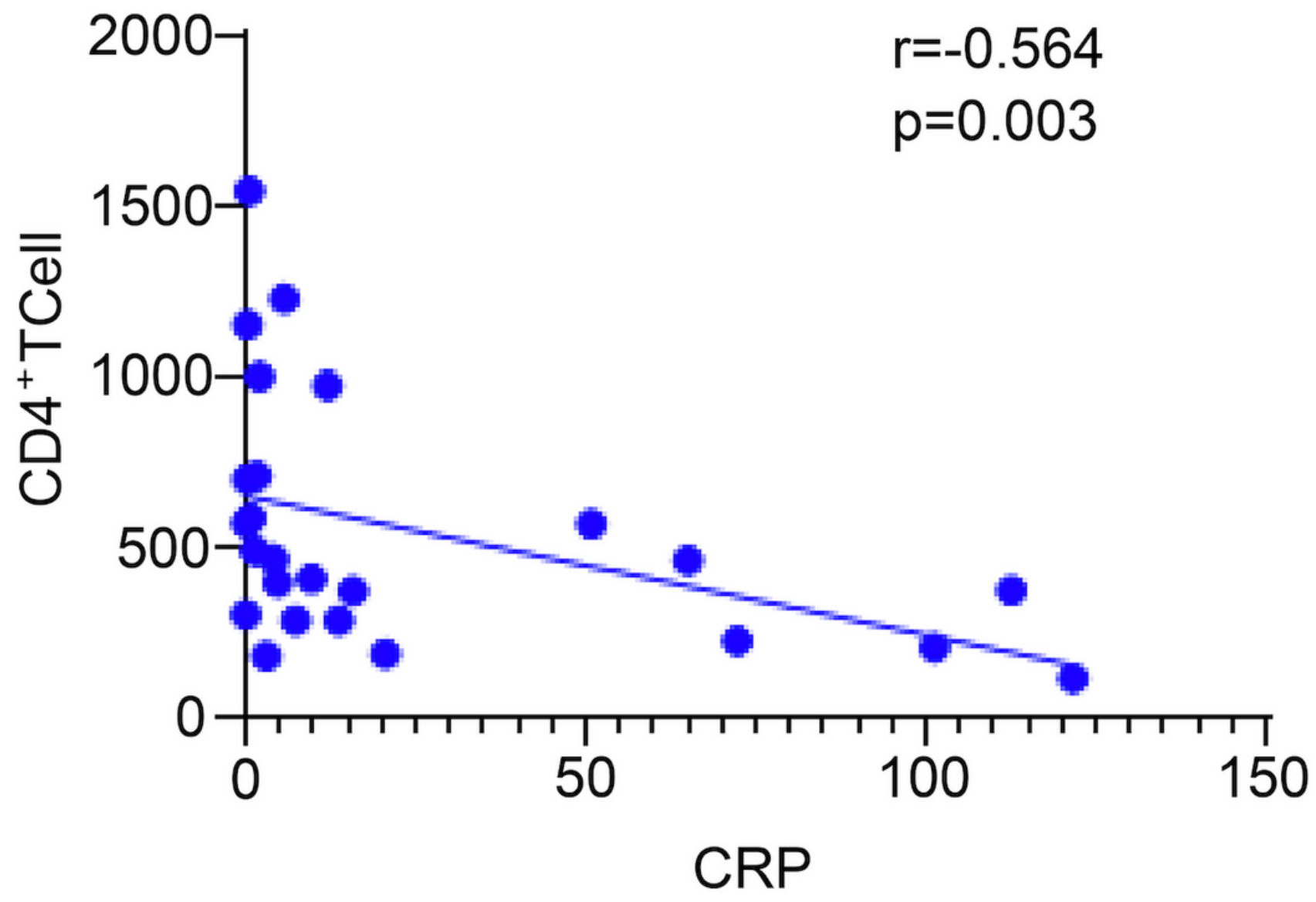

Figure 3

Correlation between CRP and CD4+T cell. 